\title{
Dermoscopy: a new perspective
}

\author{
Giuseppe Argenziano, M.D. ${ }^{1}$, Iris Zalaudek, M.D. ${ }^{2}$ \\ ${ }^{1}$ Dermatology Unit, Medical Department, Arcispedale Santa Maria Nuova, Reggio Emilia, Italy \\ ${ }^{2}$ Department of Dermatology, Medical University of Graz, Graz, Austria
}

Citation: Argenziano G, Zalaudek I. Dermoscopy: a new perspective. [editorial]. Dermatol Pract Concept 2011;1(1):12. http://dx.doi. org/10.5826/dpc.0101a12.

Copyright: @2011 Argenziano et al. This is an open-access article distributed under the terms of the Creative Commons Attribution License, which permits unrestricted use, distribution, and reproduction in any medium, provided the original author and source are credited.

Corresponding author: Giuseppe Argenziano, M.D., Dermatology Unit, Medical Department, Arcispedale Santa Maria Nuova, Reggio Emilia, Via G. Fiorelli 5, 80121 Naples, Italy. Tel. +39.336.415.093. Email: g.argenziano@gmail.com.

\section{Editorial}

It is our great pleasure to accept the invitation of the Editor-in-Chief of Dermatology Practical \& Conceptual, our friend, Harald Kittler, to write some remarks regarding the aims and scopes of the new collaboration between the International Dermoscopy Society (IDS) and Dermatology Practical \& Conceptual (DPC).

\section{The International Dermoscopy Society}

The IDS was founded in 2003 to promote clinical research in dermoscopy and to represent a clinically oriented international organization with a thrust towards helping and improving education in dermoscopy. Our membership is worldwide and currently more than 100 countries are represented, with individuals joining by direct application for free membership. Our organization conducts a world congress every three years and sister society meetings every year during the AAD congress in the USA and the EADV congress in Europe.

\section{The dermatologist's stethoscope}

By enabling visualization of anatomic structures below the epidermis, dermoscopy serves as a bridge between clinical observation and histopathology and offers clinicians an invaluable tool to detect early-stage skin cancers and resolve the diagnosis of clinically equivocal lesions.

Dermoscopy first began to gain widespread acceptance and use among dermatologists in the early 1990's. The der- matoscopes that were commercially available then were large instruments that required an oil or alcohol interface to reduce light reflection. The market has expanded greatly since that time, and instruments that are now available range from inexpensive, handheld, cross-polarized, direct contact devices (requiring no immersion oil) to complex systems with video recording.

The dermatoscope is increasingly used as the dermatologist's stethoscope, not only because it reveals a new and fascinating morphologic dimension of pigmented and nonpigmented skin tumors but also because it improves the recognition of a growing number of skin symptoms in general dermatology. Because of the growing number of dermoscopy indications, it is not a surprise that the use of this noninvasive diagnostic tool is spreading worldwide. As the number of dermoscopy users grows, the number of scientific publications on dermoscopy has also increased significantly. More than 1000 articles have been published between 2003 and 2007 , three times the number of the previous five years, and about 1100 dermoscopy articles have been referenced in PubMed just in the last $31 / 2$ years.

\section{The joint venture between the IDS and DPC}

Multiple resources are available to assist clinicians who decide to incorporate dermoscopy into their practice. The recently redesigned website of the IDS (http://www.dermoscopy-ids.org/) is an educational resource in itself, with monthly cases, tutorials, podcasts, discussion forums, recent publications, and dermoscopy-related links, including links 
to free tutorials. The recent collaboration with the Editor-inChief of DPC brings to each of the 2500+ IDS members the advantage of complimentary access to Derm101.com (http:// www.derm101.com/), the largest online library of resources for the diagnosis and treatment of skin diseases.

Moreover, DPC has become the new official organ of the IDS. The vision behind this was to give to the IDS members and to researchers the possibility to read and publish articles in the field of dermoscopy that are of higher quality. DPC has opened, in fact, a new section on dermoscopy, edited by Alon Scope, ensuring more visibility for peer-reviewed and high quality papers in the field of this useful technique. The open-access format of DPC will ensure wider readership of its scientific, including those in the dermoscopy section.

We are very confident that the collaboration between the IDS and DPC will be a success and will contribute to the continued and widespread use of dermoscopy by clinicians throughout the globe. 